\title{
AN ANALYSIS OF TRAUIMATIC BRAIN INJURY [TBI] IN TERIMS OF 3-MONTH GLASGOW OUTCOIME SCORE [GOS] AT A TERTIARY CARE CENTRE
}

KEY WORDS: Traumatic brain injury, Glasgow outcome scale, long term

\section{Dr Shrikant Govindrao Palekar Dr Kailash K Mogal Dr Vedanti Rajesh Patil Dr I Vijay Sundar*}

Neurosurgeon, Department of General surgery, Dr.Vasantrao Pawar Medical College and Hospital \& Research Centre, Adgaon, Nashik.

Department of General surgery, Dr.Vasantrao Pawar Medical College and Hospital \& Research Centre, Adgaon, Nashik.

Department of General surgery, Dr.Vasantrao Pawar Medical College and Hospital \& Research Centre, Adgaon, Nashik.

Associate professor [Neurosurgery], cancer institute [W.I.A], Adyar, chennai. *Corresponding Author

INTRODUCTION - Traumatic brain injury [TBI] most affects the working population and their earning capacity. The various sub categories of TBI in terms of clinical features, Glasgow coma scale [GCS] and radiology are well defined.We have attempted an analysis in terms of long term Glasgow outcome score [GOS] and tried to correlate with the various factors of TBI.

MATERIALS AND METHODS - All patients of TBI over 12 years and below 60 years and those without other major trauma were included over a period of about two years. The clinical features, presentation GCS, treatment given, and outcomes were assessed. The three month GOS was scored for all patients and was used to analyse the the initial data in its light.

RESULTS - A total of 200 patients were eligible for the study and were included. Of these 159 were males and 41 were females. The average age was 37.16 years. There was a relatively higher proportion of mild TBI and greater prevalence of fractures and EDH [extradural hematoma]. On analyzing with three month GOS we found that $90.4 \%$ of the patients with mild TBI had a three month GOS of 5 whereas only $31.9 \%$ of patients with moderate or severe TBI had a three month GOS of 5 .

CONCLUSION - The long term GOS is most representative of the extent to which the patient has been able to return to their pre TBI lives. In our study the three month GOS co related well with the initial GCS. Further prospective data can elaborate more on the effect of other clinical features and radiology on long term GOS.

\section{INTRODUCTION}

Traumatic brain injury [TBI] is a leading cause of morbidity and mortality around the world. One of the conundrums of traumatic brain injury is that the data is insufficient from where the incidence and socio-economic impact is the highest. We undertook a retrospective study of all traumatic brain injury patients treated at a tertiary care centre and analyzed the data in terms of epidemiological, radiological and follow up parameters.

\section{MATERIALS AND METHODS}

All patient of traumatic brain injury treated between october 2017 to december 2019 at our hospital [Dr.Vasantrao Pawar Medical College and Hospital \& Research Centre, Adgaon, Nashik] were included in the study. Preclinical data such as mode of injury, use of helmet and influence of alcohol was collected to neurological and radiological data and this was then compared with the GOS[Glasgow outcome score] at 10 days and 3 months. Inclusion criteria were all patients of head injury between age of 12 years to 60 years. Whereas the exclusion criteria were those aged below 12 years and above 60 years, those associated other major life threatening injury like severe chest trauma, severe abdominal trauma, spinal cord trauma. Associated major fracture like pelvic fracture, femur fracture, fracture of spine and cases presenting with medical cause of IC bleed. Our exclusion criteria were planned in a way to put the focus completely on TBI and its long term outcome.

\section{RESULTS}

\section{Epidemology}

A total of 200 patients were admitted with traumatic brain injury between November 2014 to November 2019. Of these 159 were males and 41 were females [gender ratio $\mathrm{M} / \mathrm{F}=$
3.87.] The average age was 37.16 years [Males-39.04 and females 36.67]. The mode of injury was road traffic accidents [RTA] in 145, fall in 23, pedestrian in 21 , and assault in 11 . Of the 145 RTA's, 142 were not wearing helmets. Alcohol intoxication was recorded in 21 patients who were involved in an RTA.

Of the 145 RTA's 3 patients were wearing helmets and although one had an EDH which required surgical evacuation all three recovered completely. The sample size of patients with helmets was too little to compare any other parameter with those not wearing helmets. Similarly of the 24 patients who were under influence of alcohol, two were initially in a poor GCS of 4 at admission and eventually expired whereas the remaining 22 recovered to a GOS of 4 or 5 at 3 months. Again the sample size being too small to make any meaningful comparison.

\section{Clinical features}

LOC [loss of consciousness] was recorded in 101 of the patients, vomiting was recorded in 61 of the patients, seizures in 14 and ENT[ear,nose or throat] bleed in 73 patients. Admission GCS[Glasgow coma scale] was 15 in 124 patients, 13 or 14 in 29 patients, between 9 to 12 in 19 patients and 8 or less in 28 patients.

\section{Radiology}

CT [computed tomography] brain was normal in 45 patients and had some finding in 155 patients, a 110 of which were RTA patients. A total of 24 patients underwent surgery. Of the 155 patients with CT finding there was no midline shift in 120 patients Midline shift was upto $5 \mathrm{~mm}$ in 17 patients, upto 10 $\mathrm{mm}$ in 15 patients and more than 10 in 3 patients. The findings were EDH [extradural hematoma] in 44 patients, 
$\mathrm{SDH}$ [subdural hematoma] in 66 , contusions in $56, \mathrm{ICH}$ [intra cerebral hematoma] in 7, fractures in 85 and others in 36 patients.

\section{Management}

Of the 24 who underwent surgery the GCS was 12 or above at admission in 15 patients, between 9 to 12 in 6 patients and less than 8 in 3 patients. Of the 24 who underwent surgery the findings were EDH in 10, SDH in 13, contusion in 9, DAI in 1 , fractures in 14 and others in 3.

Overall of the 200 patients the GCS was 15 at 10 days in 145 patients [between 12 and 15 in 172 patients]

\section{Follow up}

The GOS [Glasgow outcome score] at 10 days was 5 in 146 patients, 4 in 21 patients, 3 in 10 patients, 2 in 4 patients and 1 in 19 patients. The GOS[Glasgow outcome score] at 1 month was 5 in 151 patients, 4 in 19 patients, 3 in 6 patients, 2 in 1 patients and 1 in 23 patients. The GOS at 3 months was 5 in 157 patients, 4 in 14 patients, 3 in 4 patients, and 1 in 25 patients.

A total of 25 patients died of which 23 patients had GCS at admission of below 8 [16 patients had GCS 3 or 4], 3 of the patients were operated upon. Of the 25 who expired the mode of injury was RTA in 16, none of whom were wearing helmets, two of whom were under influence of alcohol and all of whom had LOC. Of the 25 deaths the mode of injury was pedestrian in 4 and fall in 5 patients. 3 of the 25 patients who died had been operated upon [GCS was 13,6 and 4 in each of them].

\section{Glasgow outcome score}

Analyzing the patient with good outcomes we found that of the 157 with GOS 5 at 3 months the average age was $36.49,29$ were females and 128 were males. The admission GCS was 13 and above in 142 patients and 20 of the 157 had underwent surgery. Comparing the initial GCS to the 3 month GOS we found that of the 153 patients with GCS 13 or above, 142 had GOS of 5 at 3 months and 8 had GOS of 4 at 3 months, one had a GOS of 3 and two were dead. Of the 19 patients with GCS between 9 to 12,12 had GOS of 5 at 3 months and 5 had GOS of 4 at 3 months, 2 had a GOS of 3 . Of the 28 patients with GCS 8 or below, 3 had GOS of 5 at 3 months and 1 had GOS of 4 at 3 months, one had a GOS of 3 and 23 had a GOS of 1 .

Comparing the radiological finding to the 3 month GOS and the initial GCS we found that of the 44 patients with EDH 31 were initially in GCS 13 or above, 6 were in GCS 9 to 12 and 7 were in GCS below 8. Ten of the 44 patient with EDH underwent surgery, 9 of whom had a GOS of 5 at 3 months [one had GOS of 4]. Overall three month GOS was 5 in 35 of the 44 patients with EDH, GOS was 4 in 3patients and 1 in six patients [all were deaths].

Similarly, of the 66 patients with SDH 39 were initially in GCS 13 or above, 13 were in GCS 9 to 12 and 14 were in GCS below 8. Thirteen of the 66 patient with EDH underwent surgery. Overall three month GOS was 5 in 42 in the 66 patients with $\mathrm{SDH}, \mathrm{GOS}$ was 4 in 9 patients, 3 in 1 patient and 1 in 14 patients [All were deaths].

Of the 56 patients with contusions 37 were initially in GCS 13 or above, 6 were in GCS 9 to 12 and 13 were in GCS below 8 . Nine of the 56 patient with contusions underwent surgery. Overall three month GOS was 5 in 34 in the 56 patients with contusions, GOS was 4 in 6 patients, 3 in 3 patients and 1 in 13 patients [All were deaths].

Finally of the 24 patients with DAI 17 were initially in GCS 13 or above, 3 were in GCS 9 to 12 and 4 were in GCS below 8. One of the 24 patient with DAI underwent surgery. Overall threemonth GOS was 5 in 14 in the 24 patients with DAI, GOS was 4 in 5 patients, 3 in 1 patient and 1 in 4 patients [All were deaths].

\section{DISCUSSION}

Traumatic brain injury is a massive public health problem that affects all countries. The majority of death and disability however, occur in low and middle income countries (LMICs) and the data from these countries is comparatively less. It is a paradoxical situation that the majority of published literature regarding TBI is from countries that have comparatively less volume of TBI. In many countries, the incidence of TBI due to road traffic incidents has decreased due to measures such as legislations, safer roads and helmets [1]. Another difference is that approximately $90 \%$ of the deaths due to TBI occur in LMICs [1]. As the population of India has grown, the road traffic accidents and subsequent TBIs are increasing and about one tenth of fatalities due to road traffic accidents are from India [2].

Many factors contribute to the incidence and severity of RTAs and TBI such as better awareness and obedience of traffic rules, improved legislation to discourage traffic violations and faster access to emergency care after injury. The factors that can be controlled by the health care providers are the quality of post traumatic care, standardized trauma care guidelines and effective personalized treatment. The top institutes of the country have published data showing mortality and morbidity rates similar to elsewhere in the world [3] but the same has not been replicated all over the country.

Another deficiency is the lack of data from centers which are treating high numbers of TBI patients. A systematic review of quality of TBI data from India found that after analyzing all published literature regarding TBI from India till 2015 and applying exclusion criteria, only about 20000 patients over a period of 27 years were found to have reasonably adequate data[1]. This is clearly only a small percentage of the total TBI that have occurred.We feel each tertiary hospital which treats TBI patient has to collect, arrange and publish their data, irrespective of the total number of patients. This is our humble effort with the same intention.

In our study of the 200 patients 159 were males and 41 were females which gave us a gender ratio of male to female of 3.87 which is similar to published epidemiological data [4]. As expected TBI were more common in young and middle age populations, the average age being 37.16 years [Males-39.04 and females 36.67]. The mode of injury was RTA in 145, fall in 23 , pedestrian in 21 , and assault in 11 . Of note is the fact that of the 145 RTA's, 142 were not wearing helmets which re iterates the need for stringent laws and road safety education of the population in general.

Clinically, LOC [loss of consciousness] was recorded in 101 of the patients, vomiting was recorded in 61 of the patients, seizures in 14 and ENT [ear, nose or throat] bleed in 73 patients which again is similar to other TBI data [5].

Admission GCS [Glasgow coma scale] was 15 in 124 patients, 13 or 14 in 29 patients, between 9 to 12 in 19 patients and 8 or less in 28 patients. As expected, majority of the patients [124] had mild head injuries many studies from institutions with separate trauma centers do not admit patient of mild TBI hence they are not included in the published data. In our study 47 patients [23.5\%] had moderate or severe TBI which may be explained by the relatively lesser traffic and population density of our city. Other studies which included mild TBI have reported higher percentage of moderate and severe TBI [6].

Radiologically the most common findings were SDH [subdural hematoma] in 66 patients followed by contusions in 56 patients and EDH [extradural hematoma] in 44 patients. Interestingly fracture was noted radiologically in 85 patients. Understandably the radiological findings were often overlapping with more than one finding present in one 
patient. When compared with other published data there is a variation in CT findings with some studies reporting contusions as the most common radiological finding [7] and some others reporting scalp injuries as the most common finding [8]. We did not include scalp injuries as a radiological finding due to its minimal relevance to outcome and management.

In our study due to high percentage of mild TBI only 24 patients required surgery [12\%]. Of these the GCS was 12 or above at admission in 15 patients, between 9 to 12 in 6 patients and less than 8 in 3 patients. Thought this data seems slightly different from other literature [9] it makes sense as we had a lot of fracture and EDH which are known to deteriorate over time.

In our study we did not concentrate on radiological parameters such as rotterdam scores or on initial clinical findings such anisocoria because these have well established correlations with TBI. Instead we concentrated on Glasgow outcome score [GOS] at 10 days and at 3 months as we thought that this parameter reflects most accurately the extent to which a patient of TBI has recovered and resumed his/her activities which in turn reflects the economic impact of TBI.

In our study all the patient were assessed for GOS at 10 days and 3 months. A total of 157 patients had a GOS of 5 at three months probably reflecting the large percentage of mild TBI we had initially. Of these 142 were patients of mild TBI [90.4\%], 12 had moderate TBI and 3 had severe TBI. Conversely of the 47 patients with moderate or severe TBI only 15 had GOS of five at three months [31.9\%] whereas 6 had a GOS of four, 3 had GOS of three and 23 had GOS of 1 at three months [all were cases of severe TBI].

Sinha et al had analyzed 1236 patients who underwent decompressive craniectomy [DC] for TBI, among which the follow-up GOS was assessed in 324 patients, Of these 197 had GOS of 4 or 5[10] Another study by Dhandapani et al assessed 386 patients among which 319 could be assessed for GOS at 3 months, of these 103 patients had GOS of 1 to 3 and 76 had died[11]. Although both these studies were about TBI many aspects were different from our study such as the high percentage of mild TBI and more prevalence of fractures in our study.

Of the 157 with GOS 5 at 3 months the average age was 36.49 , 29 were females and 128 were males. The admission GCS was 13 and above in 142 patients and 20 of the 157 had underwent surgery.

Overall three month GOS was 4 Or 5 in 38 of the 44 patients with EDH, in 51 of the 66 patients with SDH, 38 of the 56 patients with contusions and 18 of the 24 patients with DAI.

We feel that studies which re iterate the already proven value of radiology, initial GCS and surgical management in TBI throw less light upon the extent to which these patients of TBI are able to return to their pre trauma lifestyle.

\section{CONCLUSION}

TBI is a major cause of loss of earning power in our country because of the age group it commonly affects. This economic impact can be best studied by follow up data [Eg: GOS] which reflect how well the patients have been able to return to their pre TBI life. In our study the three month GOS co related well with the initial GCS. We need more data from every tertiary care centre treating TBI not just of the epidemiology and demographics of the affected population but also on the longterm outcomes in various sub groups of TBI.
1. Massenburg BB, Veetil DK, Raykar NP, Agrawal A, Roy N, Gerdin M. A systematic review of quantitative research on traumatic brain injury in India. Neurol India 2017;65:305-14

2. Kirankumar MR, Satri V, Satyanarayana V, Ramesh Chandra VV, Madhusudan $\mathrm{M}$, Sowjanya J.Demographic profile, clinical features, imaging and outcomes in patients with traumatic brain injury presenting to emergency room. J Clin Sci Res 2019;8:132-6.

3. Agrawal D, Ahmed S, Khan S, Gupta D, Sinha S, Satyarthee GD. Outcome in 2068 patients of head injury: Experience at a level 1 trauma centre in India. Asian J Neurosurg 2016;11:143-5.

4. Agrawal A, Munivenkatappa A, Rustagi N, Mohan PR, Subrahmanyan BV. Epidemiological characteristics affecting outcome in traumatic brain injury. J Med Soc 2017;31:28-31.

5. Gupta P, Singh J, Sharma A, Deen S, Chaudhary A, Bhansal N, et al. Epidemiological analysis and clinical characteristics of traumatic brain injuries in rural Jaipur: The first single centre experience. J Evid Based Med Healthc 2015;2:8686-91.

6. Pal R, Munivenkatappa A, Agrawal A, et al. Predicting outcome in traumatic brain injury: Sharing experience of pilot traumatic brain injury registry. International Journal of Critical Illness and Injury Science. 2016 JulSep;6(3):127-132.DOI: 10.4103/2229-5151.190650.

7. Yattoo GH, Tabish SA, Afzal WM, Kirmani A. Factors influencing outcome of head injury patients at a tertiary care teaching hospital in India. Int J Health Sci (Qassim) 2009;3:59-62.

8. Tandle RM, Keoliya AN. Patterns of head injuries in fatal road traffic accidents in a rural district of Maharashtra autopsy based study.J Indian Acad Forensic Med 2011;33:228-31.

9. Arvind K, Sanjeev L, Deepak A, Ravi R, Dogra TD. Fatal road traffic accidents and their relationship with head injuries: An epidemiological survey of five years. Indian J Neurotrauma 2008;5:63-7.

10. Sinha S, Raheja A, Garg M, Moorthy S, Agrawal D, Gupta DK, Satyarthee GD, Singh PK, Borkar SA, Gurjar H, Tandon V, Pandey RM, Sharma BS. Decompressive craniectomy in traumatic brain injury: A single-center, multivariate analysis of 1,236 patients at a tertiary care hospital in India. Neurol India 2015;63:175-83.

11. Dhandapani S, Sarda AC, Kapoor A, Salunke P, Mathuriya SN, Mukherjee KK. Validation of a new clinico-radiological grading for compound head injury: Implications on the prognosis and the need for surgical intervention. World Neurosurg 2015;84:1244-50

\section{REFERENCES}

|www.worldwidejournals.com 